Reprod. Nutr. Dévelop., 1985, 25 (2), 427-438.

\title{
Réponses endocrines à l'insuline et âge conceptionnel chez le veau
}

\author{
Elisabeth RICHET, Marie-Jeanne DAVICCO, M. DALLE (*), J.-P. BARLET \\ I.N.R.A. Theix, 63122 Ceyrat, France. \\ (*) Laboratoire de Physiologie animale, \\ Université Clermont II, 24, avenue des Landais, \\ 63170 Aubière.
}

Summary. Endocrine response to insulin and length of gestation in young calves.

The influence of hypoglycemia on pituitary and cortical and medullary adrenal response was studied in Holstein $\times$ Friesian male calves by giving an intravenous injection of bovine insulin $(0.2 \mathrm{U} / \mathrm{kg}$ b.w.). Seventeen $(9$ treated and 8 control) calves were born spontaneously at term (278 days) and 9 (5 treated and 4 control) were delivered by caesarean section on day 260 of gestation.

Insulin injections were given 1, 5, 10 and 30 days after birth. Plasma insulin and ACTH concentrations were measured by radioimmunoassay, cortisol by competitive protein binding assay, catecholamines by radio-enzymology and glucose by colorimetry (glucose oxydase).

In calves born spontaneously at term, the insulin injection induced a significant rise in plasma ACTH and cortisol concentrations on day 5 after birth; this rise occurred on days 10 and 30 after birth in calves delivered on day 260 of gestation. The rise in plasma insulin concentration following insulin injection was always more pronounced and sustained in calves delivered before term than in those born spontaneously at term. Following insulin injection, plasma epinephrine concentration increased significantly only on day 30 in calves born spontaneously at term. After treatment, no other change in plasma catecholamine concentrations was observed in any group of calves at any age.

These results indicate that the pituitary-adrenal axis responded similarly in 5-day old calves born spontaneously at term and in 10 (or 30)-day old calves delivered by caesarean section 18 days before the expected time of parturition.

Chez les veaux et les agneaux nouveau-nés, la morbidité et la mortalité les plus élevées surviennent chez les animaux de très faible poids de naissance, fréquemment nés avant terme (Ruckebusch, 1977 ; Houssin et Brelurut, 1980). Les glucocorticoïdes jouent un rôle prépondérant dans l'adaptation du nouveau-né au milieu extra-utérin, aussi bien dans la maturation des alvéoles pulmonaires par I'intermédiaire du surfactant (De Lemos et al., 1970) que dans celle des enzymes digestives (Moog, 1979). Nous avons précédemment démontré que des veaux nés spontanément entre le $252^{\mathrm{e}}$ et le $265^{\mathrm{e}} \mathrm{j}$ de gestation répondaient à la corticostimu- 
line (ACTH) injectée à l'âge de $48 \mathrm{~h}$ par une élévation de la cortisolémie moins intense et moins durable que celle observée simultanément chez des veaux de même âge, nés spontanément à terme (278 j) (Richet, Dalle et Barlet, 1982).

Dans le présent article, l'état de développement fonctionnel de l'axe hypophysosurrénalien en fonction de l'âge conceptionnel (260 à 278 j) du veau a été évalué par la mesure des concentrations sanguines en ACTH, cortisol, catécholamines en réponse à l'injection d'insuline.

\section{Matériel et méthodes.}

Animaux. - Nous avons utilisé 17 veaux mâles de génotype Holstein $\times$ Frison, nés spontanément à terme (278-280 j). N'ayant pu disposer d'animaux nés spontanément avant terme, dix veaux de même génotype ont été obtenus à la faveur d'une laparotomie effectuée sur la ligne blanche moins de 2 min après insensibilisation des vaches au pistolet d'abattage. Immédiatement après la délivrance, le cordon ombilical est ligaturé, enduit de teinture d'iode, et le veau est placé sous une couverture chauffante à $39^{\circ} \mathrm{C}$ pendant $24 \mathrm{~h}$. Malgré ces précautions la température corporelle de ces 10 veaux $\left(8 \circ^{*}\right.$ et 2 \%) s'est abaissée de $38,5^{\circ} \mathrm{C}$ à la naissance à moins de $36,5^{\circ} \mathrm{C}$ (température la plus basse observée $35,9^{\circ} \mathrm{C}$ ) dans les $3 \mathrm{~h}$ suivant la délivrance, et est remontée entre $38^{\circ} \mathrm{C}$ et $38,5^{\circ} \mathrm{C}$ $24 \mathrm{~h}$ plus tard. Cette chute de la température corporelle était associée à une baisse de $\mathrm{pH}$ sanguin de $7,28 \pm 0,10$ à $7,05 \pm 0,11$, le $\mathrm{pH}$ étant rétabli à $7,38 \pm 0,0524 \mathrm{~h}$ plus tard. Ces observations sont analogues à celles rapportées chez des veaux Frisons ou Charolais, délivrés avant terme par césarienne (Vermorel et al., 1983).

Un repas de colostrum bovin (1 I) a été offert à ces veaux 4 fois par jour le premier jour postnatal. Le même volume de colostrum (4) a été distribué en 3 et 2 repas, respectivement au cours des $2^{e}$ et $3^{e}$ jours. Grâce à ces soins, 9 des 10 veaux délivrés par césarienne ont survécu, une femelle étant morte à $7 \mathrm{j}$ de déshydratation consécutive à une diarrhée.

Après la naissance tous les veaux (délivrés par césarienne et nés spontanémenth ont été pesés et placés en cage individuelle dans une pièce à $18^{\circ} \mathrm{C}$. Ceux nés spontanément ont reçu le colostrum maternel pendant $48 \mathrm{~h}$, puis un lait reconstitué du commerce $(130 \mathrm{~g} / \mathrm{l}$ d'eau) distribué au seau 2 fois par jour (10\% du poids vif). Le même lait est distribué dans les mêmes conditions à l'âge de $4 \mathrm{j}$ aux veaux délivrés par césarienne.

Le poids de naissance des veaux délivrés par césarienne $(34,1 \pm 1,7 \mathrm{~kg})$ était plus faible que celui des animaux nés spontanément à terme $(40,6 \pm 0,9 \mathrm{~kg}$; $p<0,01)$. Pendant le premier mois postnatal le gain moyen quotidien de poids vif $(\mathrm{g})$ était plus faible chez les premiers $(387 \pm 109)$ que chez les seconds $(840 \pm 87 ; \mathrm{p}<0,01)$. Aussi, à l'âge de $30 \mathrm{j}$, le poids vif des veaux nés à terme $(64,4 \pm 2 \mathrm{~kg})$ était encore supérieur à celui des veaux délivrés par césarienne $(45,6 \pm 2,9 \mathrm{~kg} ; \mathrm{p}<0,01)$.

Prélèvements sanguins et injections. - Un cathéter est implanté dans la jugulaire droite $16 \mathrm{~h}$ avant le début de chaque expérimentation. Le sang est pré- 
levé avant l'injection et $5,10,15,20,25,30,35,40,50,60,70,80,90,120,180$ et $300 \mathrm{mn}$ plus tard, sur EDTA (14 mg d'EDTA pour $10 \mathrm{ml}$ de sang ; Donald, 1977) et un inhibiteur des peptidases (Iniprol, Laboratoires Choay, Paris) $(1 \mathrm{ml} \mathrm{d}$ 'Iniprol pour $10 \mathrm{ml}$ de sang). L'un et l'autre préviennent respectivement la formation d'agrégats de molécules d'ACTH (Godaut et Dupouy, 1981) et la dégradation enzymatique de l'hormone. Le sang est conservé dans la glace pendant moins de 5 min et centrifugé à $4{ }^{\circ} \mathrm{C}$. Le plasma est réparti pour les différents dosages et congelé à $-20^{\circ} \mathrm{C}$.

Afin d'éviter l'influence d'un éventuel rythme nycthéméral de la sécrétion des glucocorticoïdes chez les Bovins (Macadam et Eberhart, 1972 ; Thun et al., 1981), tous les traitements ont été administrés le matin à $8 h$, après un jeûne de $16 h$, grâce à un cathéter implanté dans la veine jugulaire gauche $16 \mathrm{~h}$ plus tôt. Ces injections ont été faites à l'âge de 1, 5, 10 et $30 \mathrm{j}$.

L'insuline bovine (10 mg) (Sigma ; lot $61 \mathrm{~F} 0373 ; 25,5 \mathrm{UI} / \mathrm{mg}$ ) était dissoute dans $250 \mu \mathrm{l} \mathrm{d}^{\prime} \mathrm{HCl} \mathrm{0,1} \mathrm{N}$, additionnés de $9,750 \mathrm{ml}$ de sérum physiologique, contenant $0,5 \%$ d'albumine bovine sérique. Quatorze veaux $(9$ nés à terme et 5 nés par césarienne) ont ainsi reçu par voie intraveineuse rapide $0,2 \mathrm{UI}$ d'insuline par $\mathrm{kg}$ de PV. Les 9 témoins ( 5 nés à terme et 4 délivrés par césarienne) ont reçu de la même façon le même volume de solvant $(0,1 \mathrm{ml} / \mathrm{kg} \mathrm{PV})$.

Dosages. - L'insuline est dosée par radioimmunologie à l'aide de trousses CEA (INSIK - $1 \mathrm{M}$ ). Les anticorps obtenus chez le Cobaye contre l'insuline humaine croisent parfaitement avec l'insuline bovine. La courbe de déplacement à l'équilibre obtenue avec l'insuline bovine est très voisine de la courbe standard obtenue avec I'insuline humaine. La sensibilité du dosage est de $3 \mu \mathrm{U} / \mathrm{ml}$. La précision intra- et interdosage est respectivement de $9 \%$ et de $13 \%$.

Les concentrations plasmatiques en ACTH sont déterminées grâce à un dosage radioimmunologique dérivé de celui mis au point par Donald (1977). Le dosage nécessite l'extraction préalable de l'ACTH plasmatique par l'acide silicique ( $5 \mathrm{mg} / \mathrm{ml}$ de plasma). Le rendement d'extraction est de $66 \pm 1 \%$. Les anticorps sont obtenus chez le Lapin contre I'ACTH 1-39 humaine (Laboratoires Wellcome ; lot K6051) et utilisés à une dilution finale de $1 / 70000$. Les études de parallélisme réalisées entre la courbe standard et les courbes de dilution d'extraits hypophysaires bovins ont montré que cet anticorps dirigé contre I'hormone humaine reconnaît parfaitement I'ACTH bovine. La courbe standard et le marquage hormonal ( ${ }^{125}$ l et chloramine $\mathrm{T}$, activité spécifique $450 \pm 35 \mu \mathrm{Ci} / \mathrm{g}$ ) sont réalisés en utilisant de I'ACTH 1-24 synthétique (laboratoire Ciba-Geigy). La courbe étalon obtenue avec de I'ACTH 1-39 humaine (NIAMDD - NIH) est parallèle et non statistiquement différente de celle obtenue avec I'ACTH 1-24 synthétique. L'expression en coordonnées Logit $B / B o(y)$ en fonction du logarithme de la concentration $(x)$ donne 2 droites dont les équations de régression sont les suivantes :

$$
\begin{aligned}
& y=-1,63 x+3,54 ; r=0,946 \text { pour l'ACTH } 1-24 \\
& y=-1,78 x+4,31 ; r=0,906 \text { pour l'ACTH } 1-39
\end{aligned}
$$

indiquant qu'il est préférable d'utiliser une gamme d'ACTH 1-24, donnant les valeurs les moins dispersées. La sensibilité du dosage est de $10 \mathrm{pg} / \mathrm{ml}$. La précision intra- et interdosage est respectivement de $9 \%$ et $11 \%$. Les tests de spécifi- 
cité réalisés avec le même lot d'anticorps démontrent l'absence totale de croisement avec $\alpha$-MSH et $\beta$-MSH, et un pourcentage de croisement inférieur à $1 \%$ avec $\beta$-LPH et le fragment 1-80 de la pro-opio-mélanocortine (Durand Ph., communication personnelle).

Le cortisol plasmatique est dosé par radiocompétition selon une méthode précédemment décrite (Dalle et Delost, 1976). La sensibilité du dosage est de $0,1 \mathrm{ng} / \mathrm{ml}$. La précision intra- et interdosages est respectivement de $7 \%$ et $10 \%$.

Les catécholamines plasmatiques sont dosées par radioenzymologie (Bühler et al., 1978). La sensibilité du dosage est de $10 \mathrm{pg} / \mathrm{ml}$ pour l'adrénaline, $9 \mathrm{pg} / \mathrm{ml}$ pour la noradrénaline et $14 \mathrm{pg} / \mathrm{ml}$ pour la dopamine. La précision intra- et interdosages est respectivement de $2 \%$ et $6 \%$ pour l'adrénaline, $3 \%$ et $6 \%$ pour la noradrénaline, et $10 \%$ et $7 \%$ pour la dopamine.

Le glucose plasmatique est dosé par colorimétrie à l'autoanalyseur Technicon, en utilisant la glucose-oxydase (Michel, 1971).

Les résultats sont représentés sous forme de moyenne et d'erreur standard de la moyenne. La signification statistique des différences observées a été déterminée en utilisant le test $U$ de Mann et Whitney.

\section{Résultats.}

A l'âge de $24 \mathrm{~h}$, la glycémie basale des veaux délivrés par césarienne $(0,42 \pm 0,06 \mathrm{~g} / \mathrm{l})$ est très inférieure à celle des veaux de même âge nés à terme $(1,03 \pm 0,10 \mathrm{~g} / \mathrm{l} ; \mathrm{p}<0,01)$. Cette différence a disparu à l'âge de $5 \mathrm{j}$ (délivrés par césarienne $0,92 \pm 0,04 \mathrm{~g} / \mathrm{l}$; nés à terme $1,06 \pm 0,07 \mathrm{~g} / \mathrm{l}$ ).

A tous les âges étudiés, le traitement entraîne une élévation significative de l'insulinémie, aussi bien chez les veaux nés à $260 \mathrm{j}$ que chez ceux nés à $278 \mathrm{j}$. Cependant, cette élévation est significativement plus intense et plus prolongée chez les premiers que chez les seconds (tabl. 1). L'injection d'insuline induit toujours une baisse significative de la glycémie, à l'exception de celle pratiquée à l'âge de $5 \mathrm{j}$ chez les veaux délivrés à $260 \mathrm{j}$ (tabl. 2). Chez les animaux témoins ayant reçu $0,1 \mathrm{ml} / \mathrm{kg}$ PV de sérum physiologique, l'insulinémie et la glycémie n'ont, pas présenté de variations significatives, quel que soit l'âge ou le mode de délivrance des animaux.

A l'âge de 24 h l'injection d'insuline $n$ 'induit aucune variation significative des concentrations plasmatiques d'ACTH ou de cortisol, aussi bien chez les veaux délivrés à $260 \mathrm{j}$ que chez ceux nés à $278 \mathrm{j}$. Chez ces derniers l'injection d'insuline induit une élévation transitoire mais significative des concentrations plasmatiques d'ACTH et de cortisol seulement au stade $5 j$ (tabl. 3 et 4). Chez les veaux délivrés par césarienne l'augmentation des concentrations plasmatiques d'ACTH et de cortisol est significative aux stades $10 \mathrm{j}$ et $30 \mathrm{j}$ (tabl. 3 et 4).

Chez tous les veaux, aux stades étudiés, l'hypoglycémie n'a pas entraîné de variations significatives des concentrations plasmatiques en dopamine, noradrénaline et adrénaline, à l'exception du stade $30 \mathrm{j}$ chez les veaux délivrés par césarienne. Chez ceux-ci, à cet âge, l'hypoglycémie insulinique est associée à une élévation significative de l'adrénalinémie qui passe de $118 \pm 35 \mathrm{pg} / \mathrm{ml}$ au temps 0 à $803 \pm 297 \mathrm{pg} / \mathrm{ml}(p<0,05) 30 \mathrm{~min}$ après l'injection d'insuline (fig. 1). 


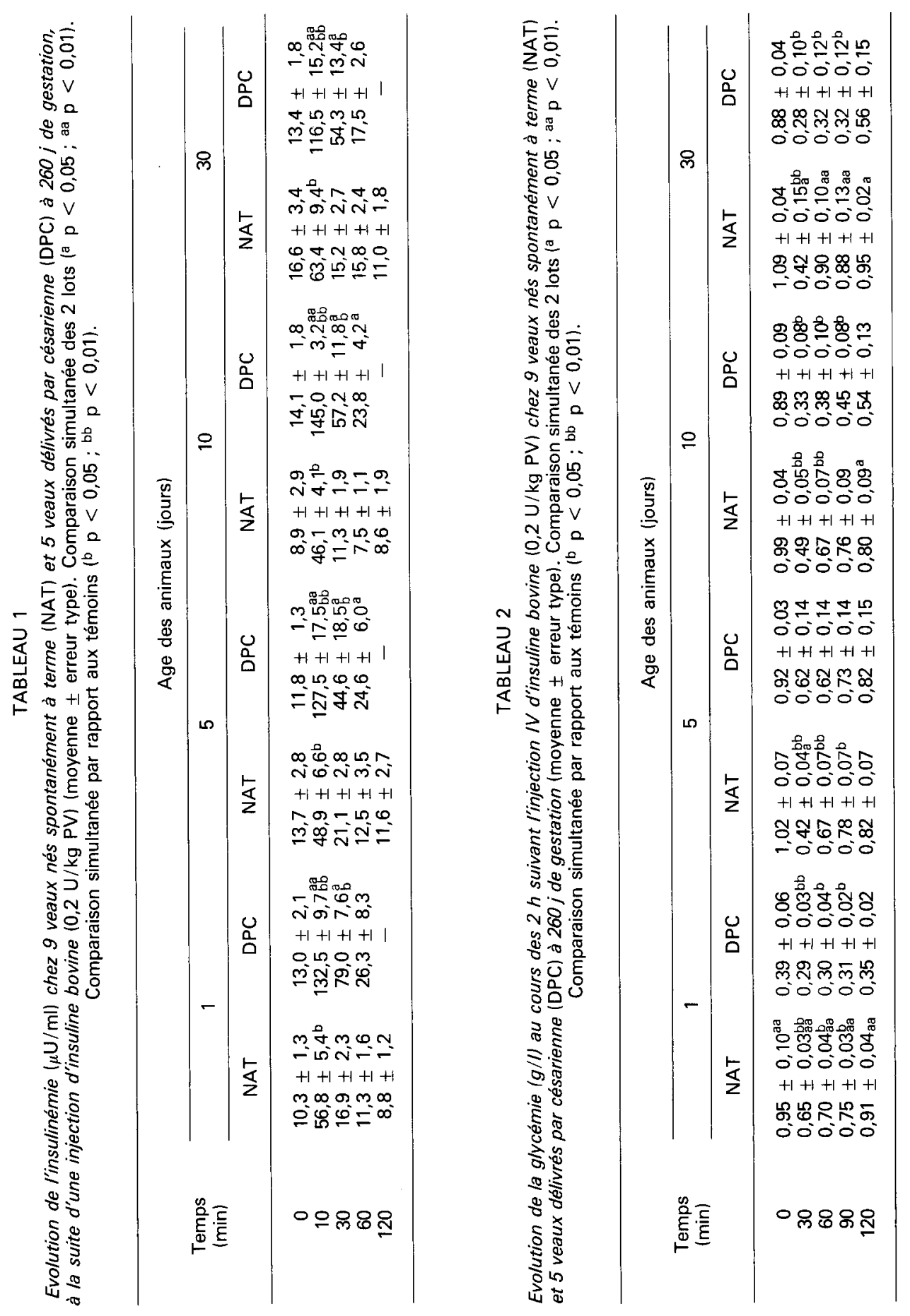



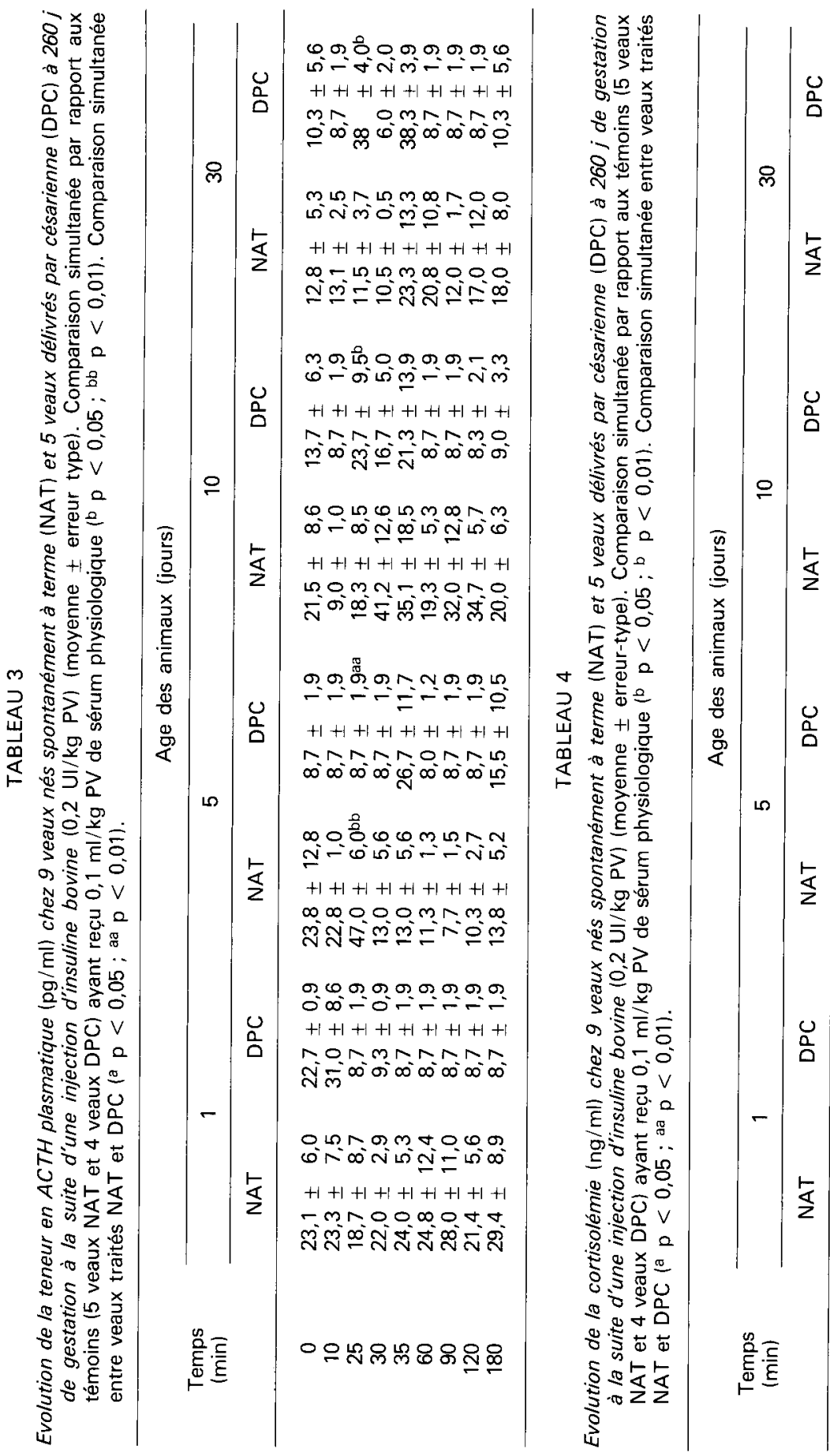

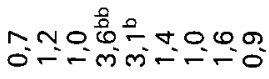

$\mathrm{H}+\mathrm{H}+\mathrm{H}+\mathrm{H}+\mathrm{H}+\mathrm{H}+\mathrm{H}+\mathrm{H}$

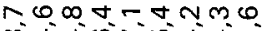

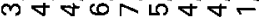

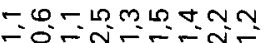

$+\mathrm{H}+\mathrm{H}+\mathrm{H}+\mathrm{H}+\mathrm{H}+\mathrm{H}+\mathrm{H}+\mathrm{H}+\mathrm{H}$

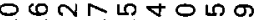
mintia

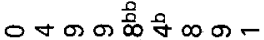
บ 心 Nóñ $+1+1+1+1+1+1+1+1+1$ $\sigma \infty$ ल $\infty \propto \sigma \infty \omega$

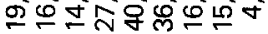

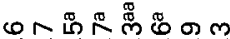
षल $+1+1+1+1+1+1+1+1$ moñọm ㅇํㄷำำ

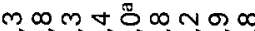

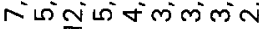

$+1+1+1+1+1+1+1+1+1$ เ 0 N $\Omega$ N $-\sigma 0$

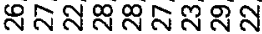

ののmの लिखि तं

$+1+1+1+1+1+1+1+1+1$

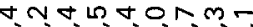

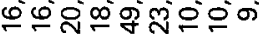

营

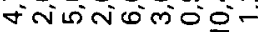

$+1+1+1+1+1+1+1+1+$ $00-\infty 0$ in $\infty \infty 0$

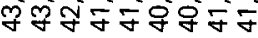

$\infty \omega 0 \forall$ Nomo

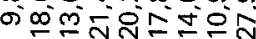
$+1+1+1+1+1+1+1+1+$

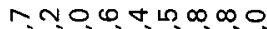

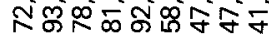

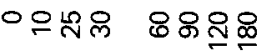




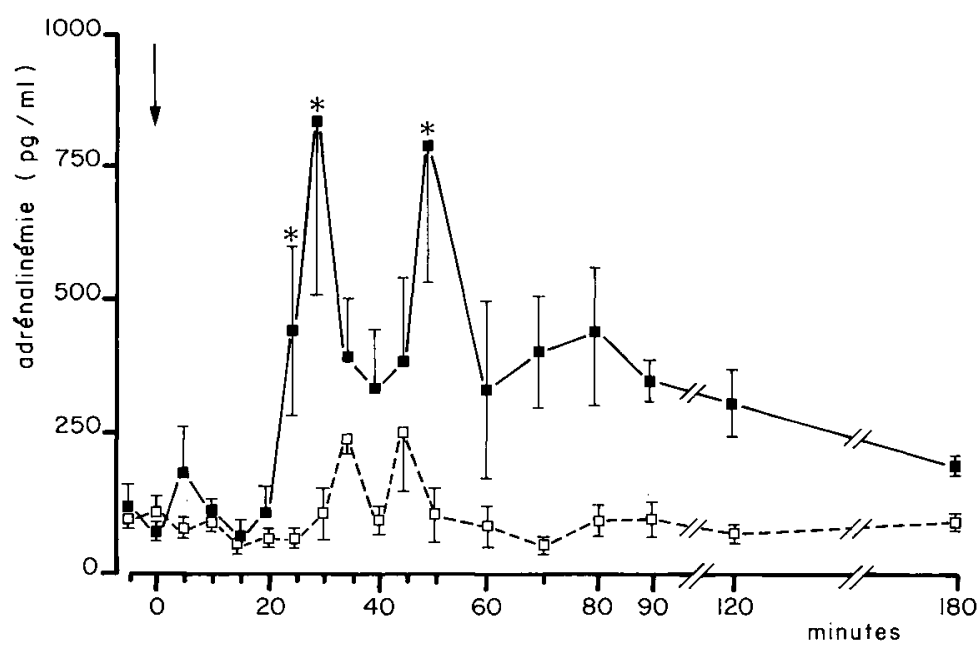

FIG. 1. - Evolution du taux plasmatique d'adrénaline au cours des $3 \mathrm{~h}$ suivant l'injection l.V. de $0,2 U / \mathrm{kg}$ P.V. d'insuline à l'âge de $30 \mathrm{j}$ chez 5 veaux délivrés par césarienne à 260 j de gestation. Les 4 veaux témoins ont reçu au même âge $0,1 \mathrm{ml}$ de sérum physiologique/kg P.V. (moyenne \pm erreur type ; ${ }^{*} P<0,05$; comparaison simultanée entre les 2 lots $d^{\prime}$ animaux : traités ( $\square \square$ ); témoins $(\square-. . \square$ ).

\section{Discussion.}

Nous avons comparé les effets sur l'hypophyse et la surrénale de l'hypoglycémie insulinique chez des veaux nés spontanément à terme et chez des veaux délivrés par césarienne avant terme. Il eût été préférable de n'utiliser que des veaux nés spontanément pour éviter l'influence éventuelle du mode de délivrance. Toutes les mises bas du troupeau expérimental ayant eu lieu à terme ou après, nous n'avons pu disposer de veaux nés spontanément avant terme, et avons dû avoir recours à des césariennes pour obtenir des veaux délivrés avant terme.

\section{Insulinémie et glycémie.}

Chez le Veau, la naissance est généralement suivie par une baisse de la glycémie basale (Daniels et al., 1974). Un des moyens les plus efficaces pour rétablir une glycémie normale est la mobilisation des réserves. Chez cet animal, avant la naissance, s'est constitué un stock important de réserves hépatiques et surtout musculaires en glycogène mobilisé grâce à l'enzyme glucose-6-phosphatase (Shelley et Neligan, 1966 ; Girard, Pintado et Ferré, 1979) mais à l'âge de 24 h, ce stock a déjà considérablement diminué (Daniels et al., 1974). L'organisme peut alors faire appel à des substrats de remplacement tels que le lactate (Comline et Edwards, 1968), produit du catabolisme du glycogène. Ce substrat permet au jeune veau d'être peu sensible à l'hypoglycémie insulinique. En effet, les signes cliniques de l'hypoglycémie sont absents tant que le lactate plasmatique est élevé (Shelley et Neligan, 1966). Or celui-ci est présent en quantités importantes chez le 
fœetus (concentration 2 à 3 fois supérieure à la concentration maternelle; Girard, Pintado et Ferré, 1979), mais il diminue au cours de la première journée suivant la naissance (Comline et Edwards, 1968). D'autres sources de substrats possibles seraient les acides gras libres et le glycérol, en particulier pour les espèces animales possédant du tissu adipeux brun (Edwards, 1971; Girard, Pintado et Ferré, 1979). C'est le cas du veau chez lequel ce tissu, présent à la naissance, disparaît progressivement au cours du premier mois postnatal (Termeulen et Molnar, 1975). II n'existe cependant pas de preuve formelle de l'intervention de ces composés dans la correction de la glycémie du Veau (Comline et Edwards, 1968).

Les veaux Hosltein $\times$ Frisons présentent un taux basal d'insuline plasmatique de l'ordre de 10 à $15 \mu \mathrm{U} / \mathrm{ml}$ (tabl. 1). L'élévation de l'insulinémie est moins intense et plus fugace chez ceux nés à terme que chez ceux délivrés par césarienne (tabl. 1). Cela peut s'expliquer par un catabolisme hormonal plus intense chez les premiers, puisque l'insulinémie basale n'est pas différente dans les deux lots (tabl. 1). Chez les Mammifères, l'insuline est dégradée au niveau de différents organes, parmi lesquels le foie joue un rôle prépondérant (Bennett et Mac Martin, 1979). Le retour à l'insulinémie basale plus rapide chez les veaux nés à terme explique que chez ceux-ci la glycémie revienne également plus rapidement à son taux initial (tabl. 2).

La glycémie des témoins est identique à celle rapportée par Bloom et al. (1975) chez des veaux Jersiais. Cependant, les animaux nés par césarienne présentent à $24 \mathrm{~h}$ une glycémie très faible dont l'origine s'explique en partie par les difficultés de récupération rencontrées par ces animaux à la naissance.

Nos résultats coïncident avec les données rencontrées dans la littérature pour ce qui est de la chronologie d'apparition du phénomène d'hypoglycémie, avec un minimum situé entre 25 et $40 \mathrm{~min}$, mais le plus souvent 30 min après l'injection d'insuline (Edwards, 1964 ; Comline et Edwards, 1968 ; Bloom et al., 1975).

Parallèlement à nos résultats, Comline et Edwards (1968) ont remarqué que le retour de la glycémie au taux basal s'effectue très lentement. Dans le cas de nos animaux, ce rétablissement a lieu au cours de la troisième heure et il est plus rapide aux stades 5 j et 10 j qu'à l'âge de $24 \mathrm{~h}$. La dose d'insuline administrée influencerait le degré d'hypoglycémie, et l'âge des animaux la durée de celle-ci (Comline et Edwards, 1968).

En ce qui concerne le comportement des veaux au cours de cette expérience, nous avons remarqué que les animaux traités, toujours en station debout pendant les premières dizaines de minutes, se couchaient au bout d'une demi-heure après I'injection. Ceci pourrait refléter un état de "fatigue générale » due à l'absence de substrat énergétique au niveau musculaire, le système nerveux central étant prioritaire en matière d'utilisation du glucose. Dans certains cas, l'intensité de I'hypoglycémie peut provoquer la manifestation de signes cliniques assez marqués, pouvant aller de la somnolence aux convulsions (Bloom et al., 1975 ; Comline et Edwards, 1968). II existe toutefois une protection des animaux jeunes envers ce phénomène. Chez le Veau recevant $1 \mathrm{U}$ d'insuline $/ \mathrm{kg} P V$, les convulsions peuvent apparaître au bout de 50 à 100 minutes à l'âge de 6 jours, alors qu'elles sont absentes chez des veaux âgés de $3 \mathrm{j}$ recevant la même dose, ceux-ci étant seulement en état de somnolence, analogue à celui observé chez la plupart 
des veaux traités. Selon Bloom et al. (1975), des veaux âgés de 2 à 5 semaines soumis à une hypoglycémie modérée (après $0,1 \mathrm{U}$ d'insuline/kg $P V$ ) ou une hypoglycémie sévère mais transitoire (après $0,5 \mathrm{U} / \mathrm{kg} P V$ ) ne manifestent aucun signe clinique, alors que la dose de $4 \mathrm{U} / \mathrm{kg} \mathrm{PV}$, qui détermine une hypoglycémie sévère et prolongée, provoque l'apparition des convulsions entre 120 et $180 \mathrm{~min}$ après l'injection d'insuline.

\section{ACTH plasmatique.}

La réponse hypophysaire à l'hypoglycémie est caractérisée par un pic plasmatique d'ACTH légèrement décalé au cours du temps selon les différentes espèces considérées (Donald, Salisbury et Nabarro, 1968 ; Goverde, 1981 ; Kellerwood, Shinsako et Dallman, 1983). Selon Bloom et al. (1975), chez le Veau, I'augmentation du taux d'ACTH après hypoglycémie insulinique est un phénomène transitoire.

A l'âge de $24 \mathrm{~h}$, chez les veaux nés à terme ou avant, l'hypoglycémie insulinique $n$ 'induit pas de variations significatives des concentrations plasmatiques d'ACTH. De même chez le Cobaye, la naissance est suivie par une période de quelques heures au cours de laquelle l'axe hypophysosurrénalien du nouveau-né est incapable de répondre à un stress psychosomatique, traduisant le fait que l'axe hypothalamohypophysosurrénalien a déjà atteint un maximum d'activité au moment de la parturition (Essono-Ondo, 1981).

Un phénomène identique a sans doute lieu dans l'espèce bovine. En effet, selon Massip (1980) l'axe hypophysocorticosurrénalien du veau nouveau-né réagit au stress du déroulement de la naissance, c'est-à-dire aux troubles occasionnés par le " travail » et en particulier à l'acidose consécutive à l'hypoxie transitoire occasionnée par la « délivrance ».

Une élévation de l'ACTH plasmatique consécutive à l'injection d'insuline est présente chez les animaux nés à terme âgés de $5 \mathrm{j}$ et disparaît à $10 \mathrm{j}$ et $30 \mathrm{j}$ (tabl. 3). Au contraire, chez les veaux nés par césarienne, la réponse hypophysaire à l'hypoglycémie n'apparaît qu'à l'âge de $10 \mathrm{j}$ et persiste à $30 \mathrm{j}$ (tabl. 3). Cette réponse retardée chez ceux-ci pourrait résulter d'une immaturité du système hypothalamohypophysosurrénalien.

\section{Cortisolémie.}

Le critère choisi pour représenter l'activité corticosurrénalienne consécutive à une injection d'insuline a été la cortisolémie. La cinétique des corticostéroïdes est parallèle à celle de l'ACTH, non superposable, mais décalée de 5 à 25 min (tabl. 4). Chez le Veau, l'axe hypothalamohypophysaire serait rapidement influencé par l'hypoglycémie mais la libération des corticostéroïdes n'est pas maintenue au-delà de $2 \mathrm{~h}$, même si I'hypoglycémie est prolongée (Bloom et al., 1975).

L'absence de réponse surrénalienne à l'hypoglycémie à l'âge de $24 \mathrm{~h}$ pourrait s'expliquer par l'absence d'élévation d'ACTH plasmatique. La chute modérée de la glycémie s'accompagne d'une élévation de la concentration plasmatique du lactate (Edwards, 1964 ; Comline et Edwards, 1968). Celui-ci intervient comme substrat, la correction de la glycémie n'implique pas d'augmentation significative 
d'ACTH et cortisol. Chez le veau nouveau-né une hypoglycémie intense induit rarement des convulsions, alors que celles-ci sont fréquentes dans les mêmes conditions à l'âge de $7 \mathrm{j}$ (Comline et Edwards, 1968). Cependant à l'âge de $10 \mathrm{j}$, les veaux nés à terme ne présentent pas d'élévation significative de la cortisolémie (tabl. 4). Ceci résulte sans doute de l'hypoglycémie modérée observée à ce stade (tabl. 2) ; chez les veaux nés à terme tout se passe comme si la réponse hypophysosurrénalienne était réglée par l'intensité de l'hypoglycémie induite par l'insuline : une réponse significative apparaît seulement à l'âge de $5 \mathrm{j}$, lorsque l'hypoglycémie est la plus intense (tabl. 3 et 4 ). Au contraire, chez le veaux délivrés par césarienne la réponse de l'hypophyse et du cortex surrénalien n'est significative qu'aux stades 10 et 30 j (tabl. 3 et 4). Ceci pourrait résulter d'une immaturité de l'hypophyse, comme semblent l'indiquer les concentrations plasmatiques, plus faibles en ACTH, mesurées chez ces animaux (tabl. 3 et 4).

La relation croissante entre la dose d'insuline administrée et les effets cliniques observés chez le Veau (Bloom et al., 1975) montre qu'il fallait utiliser une dose inférieure à $4 \mathrm{U} / \mathrm{kg} \mathrm{PV}$, dose létale chez le Veau. Le génotype du veau et/ou la nature de l'insuline injectée influent d'ailleurs sur la réponse observée. Chez 4 veaux Charolais $\times$ Frisons recevant $0,2 \mathrm{U} / \mathrm{kg}$ PV d'insuline porcine (Sigma ; lot $51 \mathrm{~F}-0467 ; 26,3 \mathrm{UI} / \mathrm{mg})$, la chute de la glycémie et l'élévation de la cortisolémie étaient plus intenses que celles observées chez les veaux $\mathrm{H} \times \mathrm{F}$ du même âge ayant reçu la même dose d'insuline bovine (Richet, 1984). L'insuline bovine et porcine diffèrent par 2 acides aminés en positions 8 et 10 sur la chaîne $A$ (Smith, 1972). II pourrait également s'agir d'une sensibilité plus grande à I'hyperinsulinémie et à l'hypoglycémie chez les veaux de ce génotype par rapport aux animaux de race laitière.

\section{Catécholamines plasmatiques.}

Le dosage des catécholamines plasmatiques au niveau d'une jugulaire externe ne donne pas une image très exacte de l'activité médullosurrénalienne. En effet la noradrénaline et surtout la dopamine présentes dans le plasma sanguin à ce niveau peuvent avoir d'autres sources (système sympathique) (Jones et Robinson, 1975).

Après hypoglycémie insulinique chez le Veau (Bloom et al., 1975) la réponse médullosurrénalienne est relativement lente. Cette sécrétion de catécholamines, bien que faible, protège au moins partiellement les veaux des convulsions pendant les situations d'hypoglycémie sévère et prolongée (Comline et Silver, 1966a, 1966b ; Edwards, 1964). Dans notre expérimentation, les veaux se trouvent dans une situation moins critique où les catécholamines n'auraient pas à intervenir, puisque leurs taux restent inchangés. L'explication est sans doute dans la présence de quantités importantes de lactate qui peut être utilisé comme substrat d'oxydation directe, réduisant ainsi l'utilisation du glucose. Par contre, à l'âge de $30 \mathrm{j}$, ce substrat est disponible en quantités réduites car les stocks de glycogène hépatique et musculaire ont considérablement diminué (Daniels et al., 1974), d'où l'intervention à ce stade des catécholamines en particulier chez les veaux délivrés par césarienne, chez lesquels l'adrénaline s'élève significativement. De plus, l'intensité de l'hypoglycémie, du fait de la dose relativement faible d'insuline utili- 
sée, a pu ne pas être suffisante pour engendrer une élévation significative des catécholamines plasmatiques. En effet, dans l'espèce humaine, une baisse de la glycémie de $0,4 \mathrm{~g} / 1$ est inefficace et c'est seulement pour une chute de $0,6 \mathrm{~g} / 1$ que l'adrénalinémie s'élève nettement (Claustre, 1978).

En conclusion, nous avons démontré que, chez des veaux nés spontanément à terme, l'hypoglycémie induite par l'insuline provoque une élévation des concentrations plasmatiques d'ACTH et de cortisol $5 \mathrm{j}$ après la naissance, alors que cette élévation apparaît seulement à $10 \mathrm{j}$, et persiste jusqu'à 30 j chez les veaux délivrés avant terme par césarienne. Ces résultats semblent démontrer une immaturité de l'axe hypothalamosurrénalien chez les animaux nés avant terme. Ceci pourrait expliquer (Munck, Guyre et Holbrook, 1984), au moins partiellement, la moins bonne résistance des veaux prématurés aux diverses agressions subies en période postnatale.

Reçu en juillet 1984.

Accepté en novembre 1984.

Remerciements. - Nous remercions Messieurs les Docteurs G. Villari et J. Y. Boidevezi (Laboratoires Ciba-Geigy) et le NIH qui nous ont fourni respectivement l'ACTH 1-24 synthétique et l'ACTH 1-39 humaine utilisée dans cette expérimentation.

\section{Références}

BENNETT H. P. J., MAC MARTIN C., 1979. Peptide hormones and their analogues : distribution clearance from the circulation, and inactivation in vivo. Pharmacol. Rev., 30, 260-292.

BLOOM S. R., EDWARDS A. V., HARDY R. N., MALINOWSKA K. M., SILVER M., 1975. Endocrine response to insulin hypoglycemia in the young calf. J. Physiol. London, 244, 783-803.

BÜHLER H. U., DA PRADA M., HEAEFELEY W., PICOTTI G. B., 1978. Plasma adrenaline, noradrenaline and dopamine in man and different animal species. J. Physiol. (London), 276, 311-320.

CLAUSTRE J., 1978. Caractères de la réponse adrénosympathique à l'hypoglycémie insulinique chez l'Homme et chez la Femme. Th. Doct. $3^{\mathrm{e}}$ cycle, Univ. Claude Bernard-Lyon I, $n^{\circ} 783$.

COMLINE R. S., EDWARDS A. V., 1968. The effects of insulin on the newborn calf. J. Physiol. London, 198, 383-404.

COMLINE R. S., SILVER M., 1966a. Development of activity in the adrenal medulla of the fetus and newborn animal. Brit. Med. Bull., 22, 16-20.

COMLINE R. S., SILVER M., 1966b. The development of the adrenal medulla of the fetal and newborn calf. J. Physiol. London, 183, 305-340.

DALLE M., DELOST P., 1976. Plasma and adrenal cortisol concentrations in fetal, newborn and mother guinea-pigs during the perinatal period. J. Endocr., 70, 207-214.

DANIELS L. B., PERKINS J. L., KRIEDER D., TUGWELL D., CARPENTIER D., 1974. Blood glucose and fructose in the newborn ruminant. J. Dairy Sci, 57, 1196-1200.

DE LEMOS R. A., SHERMETA D. W., KNELSON J. H., KOTAS R., AVERY M. E., 1970. Acceleration of appearance of pulmonary surfactant in foetal lambs by administration of corticosteroids. Ann. Rev. Respirator. Dis., 102, 459-461.

DONALD R. A., 1977. Radioimmunoassay of corticotrophin (ACTH). In : G. E. ABRAHAM, Handbook of Radioimmunoassay, 319-390, Marcel Deckker Inc., New York and Basel.

DONALD R. A., SALISBURY M. S., NABARRO J. D. N., 1968. The plasma corticotrophin response to insulin hypoglycemia, lysine-vasopressin and metyrapone in pigs. J. Endocr., 41, 509-518. 
EDWARDS A. V., 1964. Resistance to hypoglycemia in the newborn calf. J. Physiol. London, 171, 46P-47P.

EDWARDS A. V., 1971. The glycogenolytic response to stimulation of the splanchnic nerves in adrenalectomized calves, sheep, dogs, cats and pigs. J. Physiol. London, 213, 741-759.

ESSONO-ONDO A., 1981. Métabolisme du cortisol et réponse surrénalienne au stress psychosomatique au cours de la période néonatale chez le Cobaye. Th. Doct. $3^{3}$ cycle, Univ. Clermont, $n^{\circ} 667$

GIRARD J., PINTADO E., FERRÉ P., 1979. Fuel metabolism in the mammalian foetus. Ann. Biol. anim. Bioch. Biophys., 19, 181-197.

GODAUT M., DUPOUY J. P., 1981. Influence de l'héparine sur le profil d'élution chromatographique de I'ACTH, ses activités biologique et immunologique. Ann. Endocr., 41, 53C.

GOVERDE H. J. M., 1981. Bioactive versus immunoreactive adrenocorticotropin in human blood. Th. Doct. Etat, Univ. Nigmegen.

HOUSSIN Y., BRELURUT A., 1980. Mortalité avant sevrage d'agneaux de différents génotypes dans un troupeau en conduite intensive. Bull. Techn. C.R.Z.V. Theix-I.N.R.A., 40, 5-12.

JONES C.T., ROBINSON R.O., 1975. Plasma catecholamines in foetal and adult sheep. J. Physiol. London, 248, 15-33.

KELLERWOOD M. E., SHINSAKO J., DALLMAN M.F., 1983. Inhibition of the adrenocorticotropin and corticosteroid responses to hypoglycemia after prior stress. Endocrinology, 113, 491-496.

MACADAM W. R., EBERHARDT R. J., 1972. Diurnal variation in plasma corticosteroids concentration in dairy cattle. J. Dairy Sci., 55, 1792-1795.

MASSIP A., 1980. Relationship between $\mathrm{pH}$, plasma cortisol and glucose concentrations in the calf at birth. Br. Vet. J., 136, 597-601.

MICHEL M. C., 1971. Analyse quantitative de quelques substances azotées et glucidiques en milieu biologique. Essai de rationalisation. Th. Doct. Univ., Université Clermont.

MOOG F., 1979. Endocrine influences on the functional differenciation of the small intestine. $J$. anim. Sci., 49, 239-249.

MUNCK A., GUYRE P. M., HOLBROOK N. J., 1984. Physiological functions of glucocorticoids in stress and their relation to pharmacological actions. Endocrine Rev., 5, 25-44.

RICHET E., 1984. La fonction surrénalienne du Veau au cours de la période périnatale. Thèse Doctorat $3^{e}$ cycle, Université Clermont, $\mathrm{n}^{\circ} 767$.

RICHET E., DALLE M., BARLET J.-P., 1982. Réponse du jeune veau à ACTH. Influence de la durée de gestation. J. Physiol. Paris, 78, 21A.

RUCKEBUSCH Y., 1977. Physiologie néonatale, 91-98. In MORNET P., ESPINASSE J. Le Veau. Anatomie. Physiologie. Elevage. Alimentation. Production. Pathologie, Maloine, Paris.

SHELLEY H. J., NELIGAN G. A., 1966. Neonatal hypoglycemia. Brit. Med. Bull., 22, 34-39.

SMITH L. F., 1972. Amino acid sequences of insulins. Diabetes, 21 (supplt 2), 457-460.

TERMEULEN U., MOLNAR S., 1975. Untersuchungen zur Morphologie und Physiologie des perirenalen Fettgewebes beim Kalb und der Einfluss der Ungebungstemperature auf seine Funktion. Z. Tierphysiol. Tierernährg u. Futtermittelkde, 35, 243-252.

THUN R., EGGENBERGER E., ZEROBIN K., LUSHER T., VETTER W., 1981. Twenty-four hour secretory pattern in the bull. Evidence of episodic secretion and circadian rythm. Endocrinology, 109, 2208-2212.

VERMOREL M., DARDILLAT C., VERNET J., SAIDO, DEMIGNÉ C., 1983. Energy metabolism and thermoregulation in the newborn calf. Ann. Rech. Vét., 14, 382-389. 